\title{
ENSINO DE ODONTOLOGIA HOSPITALAR NO CURSO DE ODONTOLOGIA NA REGIÃO NORDESTE DO BRASIL
}

\author{
HOSPITAL ODONTOLOGY TEACHING IN THE DENTISTRY COURSE \\ IN THE NORTHEAST REGION OF BRAZIL
}

\author{
Julia Tavares Palmeira ${ }^{1}$ \\ Regina Mendes da Silva ${ }^{2}$ \\ José Henrique de Araújo Cruz ${ }^{3}$ \\ Itamar da Silva Nunes ${ }^{4}$ \\ Manuella Santos Carneiro Almeida ${ }^{5}$ \\ Camila Helena Machado da Costa Figueiredo ${ }^{6}$
}

RESUMO: OBJETIVO: O objetivo desse trabalho verificou a presença da disciplina de odontologia hospitalar na grade curricular dos cursos de odontologia nas universidades da região Nordeste do Brasil. MÉTODOS: Este estudo foi do tipo descritivo, que adotou como estratégia de coleta de dados a consulta de dados disponíveis no site do Ministério da Educação (MEC) e no Conselho Federal de Odontologia (CFO), por meio da visita dos sites dos cursos de odontologia nas universidades da região Nordeste do Brasil. Foram incluídas na pesquisa todas as universidades que oferecem o curso de odontologia cadastrados no MEC ou no CFO, sendo instituições públicas ou privadas da região Nordeste do País. E foram excluídas da amostra todas as instituições de ensino superior que não disponibilizavam os seus componentes curriculares nos seus respectivos sites. $O$ universo foi composto por 95 universidades, contudo, após a aplicação dos critérios de inclusão e exclusão obteve-se uma amostra de 86 faculdades que dispuseram a grade curricular nos sites correspondentes. Os dados coletados foram registrados na forma de banco de dados do programa de informática SPSS (Statistical Package for

\footnotetext{
${ }^{1}$ Acadêmica do Curso de Odontologia da Universidade Federal de Campina Grande-Paraíba, Brasil. E-mail: julia.palmeira@hotmail.com.

${ }^{2}$ Acadêmica do Curso de Odontologia da Universidade Federal de Campina Grande-Paraíba, Brasil. E-mail: reginamendes16@hotmail.com.

${ }^{3}$ Cirurgião Dentista formado na Universidade Federal de Campina Grande-Paraíba, Brasil. E-mail: henrique_araujo1992@hotmail.com.

${ }^{4}$ Cirurgião Dentista formado na Universidade Federal de Campina Grande-Paraíba, Brasil. E-mail: itamarodontoufcg@gmail.com.

${ }^{5}$ Professora Doutor do Curso de Odontologia da Universidade Federal de Campina Grande-Paraíba, Brasil. E-mail: manuellacarneiro@hotmail.com.

${ }^{6}$ Professora Doutor do Curso de Odontologia da Universidade Federal de Campina Grande-Paraíba, Brasil. E-mail: camila_helena_@hotmail.com.
} 
Social Sciences) para Windows, versão 13.0, e foram trabalhados pela descritiva. RESULTADOS: Das 86 faculdades consultadas, $16(18,60 \%)$ apresentaram a disciplina de odontologia hospitalar, sendo $10(62,5 \%)$ em universidade particular e as outras $6(37,5 \%)$ em universidade pública. As outras 70 universidades $(81,39 \%)$ não apresentaram a disciplina. CONCLUSÃO: Mediante os resultados obtidos, pode-se concluir que o número de centros acadêmicos que dispõem da disciplina em análise, é extremamente escasso.

Palavras chave: Hospital; Educação em Odontologia; Integração Curricular.

ABSTRACT: OBJECTIVE: The objective of this study was to verify the presence of the discipline of hospital dentistry in the curriculum of dentistry courses at universities in the Northeast region of Brazil. METHODS: This was a descriptive study, adopting as data collection strategy the consultation of data available on the website of the Ministry of Education (MEC) and the Federal Council of Dentistry (CFO), by visiting the websites of dental courses. in the universities of the Northeast region of Brazil. The study included all universities that offer dental courses registered at MEC or CFO, being public or private institutions in the Northeast of the country. And all higher education institutions that did not provide their curriculum components in the sample were excluded from the sample. their respective websites. The universe was made up of 95 universities, however, after applying the inclusion and exclusion criteria, we obtained a sample of 86 colleges that arranged the curriculum in the corresponding sites. The data collected were recorded as a database of the computer program SPSS (Statistical Package for Social Sciences) for Windows, version 13.0, and were worked through the descriptive. RESULTS: Of the 86 colleges consulted, 16 (18.60\%) presented the discipline of hospital dentistry, 10 $(62.5 \%)$ in a private university and the other $6(37.5 \%)$ in a public university. The other 70 universities (81.39\%) did not present the discipline. CONCLUSION: From the results obtained, it can be concluded that the number of academic centers that have the subject under analysis is extremely scarce.

Keywords: Hospital; Dentistry education; Curriculum Integration. 


\section{INTRODUÇÃO}

As primeiras evidências acerca da relação entre alterações orais e comprometimento sistêmico são de 2.100 a.C. Desde então, frequentemente se tem pesquisado e relatado sobre como a condição bucal influencia na evolução e resposta de condições sistêmicas, assim como a saúde bucal pode ser alterada pelas alterações sistêmicas presentes no paciente (ROCHA et al., 2014).

A Odontologia Hospitalar consiste em um conjunto de práticas de baixa, média ou alta complexidade, que tratam e previnem enfermidades por meio de procedimentos em âmbito hospitalar cujo foco principal é o cuidado de pacientes críticos que necessitam de tratamentos especiais (SALDANHA et al., 2015). A odontologia hospitalar cuida das alterações orais que requerem ação de equipes multidisciplinares de alta complexidade ao paciente. Contudo, a higienização bucal dos pacientes internados é por vezes negligenciada, não só por parte da equipe de enfermagem, mas também dos acompanhantes. O problema é intensificado pela falta de integralidade no atendimento do paciente, um fator muito comum na maioria dos hospitais (LAGES et al., 2014).

É conhecido que orientações e práticas de higiene oral em âmbito hospitalar proporcionam bem-estar, melhoria da autoestima, aumenta a qualidade de vida e auxiliam na prevenção da formação e do desenvolvimento de biofilme e, sobretudo, diminuição do risco de infecções provenientes da microbiota bucal (FERNANDES et al., 2016). Em ambiente de terapia intensiva é constante a vigilância da infecção por toda a equipe da UTI e pelo CCIH - Comissão de Controle de Infecção Hospitalar. Considerando-se que mais da metade das bactérias do organismo encontra-se na cavidade oral e sua descontaminação diminui o risco de pneumonia nosocomial, PAV, endocardite infecciosa e SEPSE, fundamenta-se a vigilância da cavidade oral pelo cirurgião-dentista na UTI (LOBÃO et al., 2016).

É de suma importância a presença de um cirurgião-dentista dentro do hospital sendo esta pautada no conhecimento de que uma adequada saúde bucal pode 
influenciar positivamente o desfecho clínico do paciente, reduzindo ou até mesmo evitando fatores que possam contribuir negativamente no tratamento sistêmico do mesmo (GONÇALVES et al., 2014). Sabe-se que a formação do cirurgião dentista deve ser pautada na construção de habilidades necessárias para um bom desempenho profissional em suas tomadas de decisões. Tendo isso em mente, a integração curricular da disciplina de odontologia hospitalar, surge como um promissor meio de progredir nessa proposta (LINO-JÚNIOR et al., 2015).

De acordo com o artigo 26 do Código de Ética Odontológica, referente à Odontologia Hospitalar (capítulo X), é dever do cirurgião-dentista internar e assistir pacientes em hospitais públicos e privados, com ou sem caráter filantrópico, respeitadas as normas técnicos-administrativas das instituições (DANTAS et al., 2015).

O objetivo desse trabalho verificou a presença da disciplina de odontologia hospitalar na grade curricular dos cursos de odontologia nas universidades da região Nordeste do Brasil.

\section{MÉTODO}

Este estudo foi do tipo descritivo, sendo a coleta de dados feita a partir da consulta de dados disponíveis no site do Ministério da Educação (MEC) e no Conselho Federal de Odontologia (CFO), por meio do acesso aos sites dos cursos de odontologia nas universidades da região Nordeste do Brasil. Foram incluídas na pesquisa todas as universidades que oferecem o curso de odontologia cadastrados no MEC ou no CFO, sendo instituições públicas ou privadas da região Nordeste do País. E foram excluídas da amostra todas as instituições de ensino superior que não disponibilizavam os seus componentes curriculares nos seus respectivos sites.

O universo foi composto por 95 universidades, contudo, após a aplicação dos critérios de inclusão, obteve-se uma amostra de 86 faculdades que dispuseram a grade curricular nos sites correspondentes. 
A coleta de dados foi realizada por um pesquisador, que acessou o site do MEC e CFO nos quais foram arquivados os nomes das universidades, os sites e os e-mails das IESs (Instituições de ensino Superior) que ofertam o curso de Odontologia na região Nordeste em uma tabela.

De posse desses dados, o pesquisador localizou nos sites das IESs as matrizes curriculares, verificou a presença ou ausência da disciplina de odontologia hospitalar na grade curricular ou no plano de curso (PPC) e se esta era obrigatória ou optativa, também foi verificada a carga horária da disciplina. Os dados coletados foram registrados na forma de banco de dados do programa de informática Statistical Package for Social Sciences (SPSS) para Windows, versão 13.0, e foram trabalhados pela análise descritiva absoluta e relativa dos dados.

\section{RESULTADOS}

A tabela 1 aponta o número de faculdades públicas e privadas da região Nordeste que fornecem a disciplina de Odontologia Hospitalar.

Tabela 1: Total de faculdades públicas e privadas da região Nordeste que fornecem a disciplina de Odontologia Hospitalar - 2019.

\begin{tabular}{lccc}
\hline \multicolumn{1}{c}{ ESTADOS } & Particular & Pública & Total por estado \\
\hline Alagoas & 1 & 0 & 5 \\
\hline Bahia & 2 & 2 & 25 \\
\hline Ceará & 1 & 0 & 11 \\
\hline Maranhão & 3 & 0 & 8 \\
\hline Paraíba & 2 & 2 & 12 \\
\hline Pernambuco & 0 & 0 & 9 \\
\hline Piauí & 0 & 1 & 7 \\
\hline Rio Grande do Norte & 0 & 1 & 5 \\
\hline Sergipe & 1 & 0 & 4 \\
\hline TOTAL & 10 & 6 & 86 \\
\hline Fonte: Autara propria
\end{tabular}

Fonte: Autoria própria 
Das 86 faculdades consultadas, 16 (18,60\%) apresentaram a disciplina de odontologia hospitalar, sendo 10 (62,5\%) em universidade particular e as outras 6 $(37,5 \%)$ em universidade pública. As outras 70 universidades $(81,39 \%)$ não apresentaram a disciplina.

As instituições de ensino superior que ofertaram da disciplina em análise, 13 $(81,25 \%)$ são do tipo obrigatória e apenas $3(18,75 \%)$ são do tipo optativa. Sendo que as cargas horárias variaram de 20 horas por semestre na Faculdade Pitágoras São Luís no Maranhão a 85 horas na Universidade Federal da Bahia (UFBA). A carga horária citada com mais frequência foi correspondente a 60 horas $(28,57 \%)$, retirou-se a Faculdade de Tecnologia de Alagoas $(A L)$ e as Faculdades Integradas de Patos - UNIFIP (PB) da amostra pois não possuíam em seus respectivos sites a carga horária (Tabela 2).

Tabela 2: Divisão das faculdades (Pública/Privada) que possuem a disciplina de Odontologia Hospitalar (Optativa/Obrigatória) e suas respectivas cargas horárias 2019.

\begin{tabular}{|c|c|c|c|}
\hline Instituição de Ensino Superior & $\begin{array}{l}\text { Públical } \\
\text { Privada }\end{array}$ & $\begin{array}{c}\text { Optativa/ } \\
\text { Obrigatória }\end{array}$ & $\begin{array}{l}\text { Carga } \\
\text { Horária }\end{array}$ \\
\hline Faculdade de Tecnologia de Alagoas (AL) & Privada & Obrigatória & - \\
\hline Universidade Federal da Bahia UFBA (BA) & Pública & Optativa & $85 \mathrm{hrs}$ \\
\hline $\begin{array}{l}\text { Universidade Estadual de Feira de Santana } \\
\text { UEFS (BA) }\end{array}$ & Pública & Obrigatória & $30 \mathrm{hrs}$ \\
\hline $\begin{array}{l}\text { Faculdade de Ensino Superior da Cidade de } \\
\text { Feira de Santana (BA) }\end{array}$ & Privada & Obrigatória & $80 \mathrm{hrs}$ \\
\hline $\begin{array}{l}\text { Escola Bahiana de Medicina e Saúde Pública } \\
\text { EBMSP (BA) }\end{array}$ & Privada & Obrigatória & $72 \mathrm{hrs}$ \\
\hline $\begin{array}{l}\text { Instituto Superior de Teologia Aplicada - INTA } \\
(\mathrm{CE})\end{array}$ & Privada & Obrigatória & $60 \mathrm{hrs}$ \\
\hline $\begin{array}{l}\text { Unidade de Ensino Dom Bosco UNDB São Luís } \\
\text { (MA) }\end{array}$ & Particular & Obrigatória & $68 \mathrm{hrs}$ \\
\hline Faculdade Pitágoras São Luís & ular & tória & $20 \mathrm{hrs}$ \\
\hline Faculdade Pitágoras - Imperatriz (MA) & Particular & Obrigatória & $60 \mathrm{hrs}$ \\
\hline Universidade Federal da Paraíba (PB) & Pública & Obrigatória & $70 \mathrm{hrs}$ \\
\hline Universidade Estadual da Paraíba (PB) & Pública & Optativa & $30 \mathrm{hrs}$ \\
\hline Faculdades Integradas de Patos UNIFIP (PB) & Particular & Obrigatória & - \\
\hline $\begin{array}{l}\text { Instituto de Educação Superior da Paraíba } \\
\text { IESP (PB) }\end{array}$ & $\mathrm{P}$ & Obr & $40 \mathrm{~h}$ \\
\hline Universidade Estadual do Piauí - UESPI (PI) & Pública & Obrigatória & $60 \mathrm{hrs}$ \\
\hline
\end{tabular}




\begin{tabular}{lccc}
\hline $\begin{array}{l}\text { Universidade Federal do Rio Grande do Norte } \\
\text { (RN) }\end{array}$ & Pública & Optativa & $60 \mathrm{hrs}$ \\
\hline Universidade Tiradentes - Unit (SE) & Particular & Obrigatória & $40 \mathrm{hrs}$ \\
\hline
\end{tabular}

Fonte: Autoria própria.

Das faculdades 16 listadas na tabela acima, apenas 12 constavam nos respectivos sites o período em que a disciplina era ofertada, sendo no décimo período a maior ocorrência $5(42 \%)$ e a menor no terceiro e no nono período respectivamente $(8 \%)$.

Gráfico 1: Distribuição por períodos cuja disciplina é ofertada nas faculdades que a possuem - 2019.

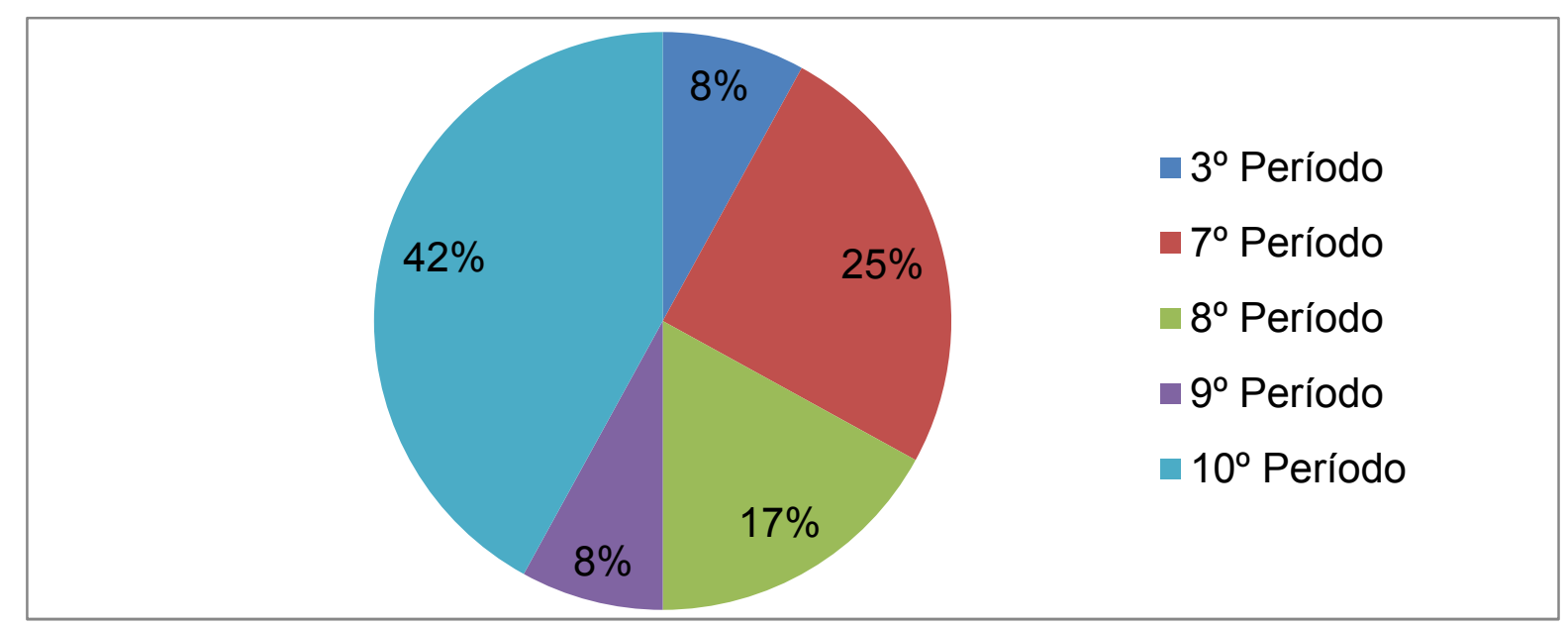

Fonte: Autoria própria

\section{DISCUSSÃO}

As complexidades do mundo contemporâneo aliado aos avanços tecnológicos e científicos exigem que o conhecimento seja trabalhado, nas instituições de ensino, de forma multidimensional. Para a formação profissional em saúde, especialmente em odontologia, isto significa a necessidade de ampliação dos ambientes educacionais, das técnicas educativas, vivência com reais situações profissionais e com diferentes graus de complexidade (OGAWA et al., 2015). Uma compreensão 
biopsicossocial do processo saúde-doença-cuidado tem permitido ampliar a visão sobre a formação profissional, evidenciando não apenas a necessidade de se adquirir conhecimentos teóricos e técnicos interdisciplinares, como também de se criar mecanismos para o profissional pensar enquanto sujeito implicado no processo de cuidado (GAETTI-JARDIM et al., 2013).

Segundo Gaetti-Jardim et al. (2013) para o cirurgião-dentista é um desafio atuar em hospitais, pois faz com que os dentistas saiam de sua zona de conforto, representada por procedimentos simples, em pacientes saudáveis ou ligeiramente comprometidos, em consultórios confortáveis, ergonômicos e bem planejados. Em âmbito hospitalar esta questão diverge muito comparando à rotina de um consultório odontológico, isso se explica ao fato de que no hospital os procedimentos são mais complexos. Portanto é muito importante essa comunicação multidisciplinar para proporcionar uma boa estadia ao paciente internado (FONSECA, 2012).

Wayama et al. (2014) afirmam que é extremamente válido para o graduando, como um futuro cirurgião-dentista durante a sua formação acadêmica, o seu incentivo e preparo para manejo do paciente hospitalizado. Enfatizam com isso, a importância de faculdades de Odontologia abordarem este tema em suas grades curriculares (PINHEIRO et al., 2014). Um estudo realizado em 2014 com formandos, tendo o objetivo de avaliar os conhecimentos acerca da Odontologia Hospitalar, revelou conhecimentos confusos sobre a área, certo desconhecimento quanto aos procedimentos e patologias adquiridas em hospitais, como a pneumonia nosocomial, e ainda, alguns entrevistados manifestaram não ter segurança para atuar no tratamento de pacientes em unidades de terapia intensiva. Evidentemente, mais de $65 \%$ deles não apresentaram interesse em atuar na área depois de formados (CONRADO, 2014).

Uma das maneiras encontradas para adequar as práticas e a atuação dos cirurgiões-dentistas em ambientes hospitalares encontra-se relacionada com a oferta de cursos em nível de habilitação e residências odontológicas. Entretanto, em termos de graduação em Odontologia, a presença do componente curricular de Odontologia Hospitalar nos currículos das instituições de ensino superior ainda se encontra bastante discreta. Neste contexto, destaca-se a importância dos estudantes de graduação em Odontologia, como futuros cirurgiões-dentistas, serem 
incentivados e preparados, durante a sua formação acadêmica, para o manejo adequado do paciente em nível hospitalar (GODOl et al., 2009).

Em razão da falsa crença que o cirurgião-dentista não precisa atuar em ambiente hospitalar, a raiz do problema se inicia ainda na graduação. A disciplina odontologia hospitalar no currículo de graduação das faculdades de Odontologia é pouco abordada, em alguns casos este tema é abordado somente em nível de especialização. Em todas as faculdades triadas da região nordeste, apenas 16 $(18,60 \%)$ possuem a disciplina.

Quanto maior a carga horária de uma disciplina, maiores são as chances de adquirir um maior grau de conhecimento de forma mais abrangente. Mesmo compreendendo toda a importância do conhecimento em análise tanto na teoria quanto na prática, ainda existem faculdades que abordam a temática de maneira diligente. Faculdades Pitágoras (São Luís) e a Universidade estadual da Paraíba correspondem a carga horária de 20 horas e 30 horas, respectivamente.

Mediante dos imensuráveis e promissores resultados proporcionados pela odontologia hospitalar, ainda existe estado que em todas as faculdades consultadas, nenhuma possuiu a disciplina. A localidade em questão é Pernambuco, possuindo ausência da ciência em todas as $9(10,46 \%)$ academias analisadas.

A discussão a respeito da inserção desse componente curricular nas matrizes curriculares dos cursos de Odontologia deve ocorrer com o intuito de aumentar a participação e a atuação dos cirurgiões-dentistas no ambiente hospitalar, mostrando aos estudantes e à população a importância de melhorar o atendimento dos pacientes hospitalizados e a necessidade de uma formação profissional alicerçada nos princípios de integralidade e multiprofissionalismo (DORO et al., 2009).

Mesmo com toda sua importância e a urgência de capacitação dos acadêmicos para esse novo cenário da odontologia contemporânea, o número de faculdades públicas que dispõem desse conhecimento, se apresentou totalmente insignificativo. De todas as 16 faculdades públicas que apresentaram o curso de odontologia hospitalar, apenas 6 (37,5\%) ofertam a disciplina em estudo.

É possível notar que não existe um padrão na disponibilidade por período das disciplinas. Mesmo sendo uma temática que se torna necessária agregar conhecimento de disciplinas pré-existentes, existe uma faculdade que aborda o 
assunto precocemente, no terceiro período correspondendo ao total de $8 \%$ do valor total da amostra.

Por a ciência ser mais do que necessária no cenário hospitalar, além de ser presente em todas as universidades, deveria ser parte indispensável da formação profissional. Mesmo que a maioria das universidades tenha esse conhecimento no regimento de obrigatoriedade $(81,25 \%)$, ainda existem faculdades que oferecem como disciplina eletiva (18,75\%). A consequência dessa questão, é que o acadêmico pode ter a possibilidade de não obter esse conhecimento dentro do ambiente universitário e comprometendo dessa forma, sua atuação no âmbito hospitalar, tendo que recorrer a formações extra acadêmicas para obter tal conhecimento.

Uma das limitações do estudo, seria a criação crescente de cursos de odontologia periodicamente. Com uma taxa exponencial de abertura de cursos de odontologia todos os anos, dificulta a contabilização exata ao longo de um ano, sendo necessário novos estudos para acompanhar as estimativas.

\section{CONCLUSÃO}

Pode-se concluir que o número de centros acadêmicos que dispõem da disciplina em análise, é extremamente escasso. Com base no cenário atual da Odontologia Hospitalar no Brasil, o acesso à informação e ao conhecimento, quanto ao atendimento e abordagens específicas a esse público alvo, vem crescendo, mas ainda necessita se disseminar e adquirir espaço e apoio significativos. 


\section{REFERÊNCIAS BIBLIOGRÁFICAS}

BRASIL. Ministério da Saúde. Ministério da Educação. Portaria interministerial $n^{\circ} 2.101$, de 3 de novembro de 2005. Institui o Programa Nacional de Reorientação da Formação Profissional em Saúde - Pró-Saúde - para os cursos de Graduação em Medicina, Enfermagem e Odontologia. Diário Oficial da União 2005; nov. 4.

CONRADO, C. A; GOMES, G. S; ROBAZZA, C. R. C. O projeto pedagógico: estruturação e desenvolvimento curriculares - o currículo multidisciplinar integrado. In: Terada RSS, Nakama L. A implantação das Diretrizes Curriculares Nacionais de Odontologia: a experiência de Maringá. São Paulo: Hucitec. J. Manag Prim Health care. v.3, n.2, p.158-178, 2014.

CONSELHO FEDERAL DE ODONTOLOGIA. Resolução $n^{\circ}$. 163, de 09 de novembro de 2015. Conceitua a Odontologia Hospitalar e define a atuação do cirurgião-dentista habilitado a exercêla. 2015.

DANTAS, B.O., De ARAÚJO, I.A., De ARAÚJO, H.B.N, De ARAÚJO, E.C., BEZERRA, A.C.B., MIRANDA, A.F. Saúde bucal e cuidados na Unidade de Terapia Intensiva. R Odontol Planal Cent. v.5, n.1, p.28-32, 2015.

DORO, G.M.; FIALHO, L.M.; LOSEKANN, M.; PFEIFF, D.N. Projeto "Odontologia Hospitalar". Rev ABENO. V.6, n.1, p.49-53, 2006.

FERNANDES, A.S.; EMILIANO, G.B.G.; MARTINS, A.R.L.A.; SOUZA, G.C.A. Conhecimentos e práticas de saúde bucal por pacientes internados e equipe hospitalar. Revista Ciência Plural. V.2, n.3, p.03-16, 2016.

FONSECA, E.P. The National Curriculum Guidelines and training of Brazilian dentists. J Manag Prim Health Care. v.3, n.2, p. 158-178, 2012.

GAETTI-JARDIM ,E.C.; SETTI, J.S.; CHEADE, M.F.M.; MENDONÇA, J.C.G. Atenção odontológica a pacientes hospitalizados: revisão da literatura e proposta de protocolo de higiene oral. Rev bras ciênc saúde. v.11, n.35, p.31-36, 2013.

GODOI, A.P.T.; FRANCESCO, A.R.; DUARTE, A.; KEMP, A.P.T.; SILVA-LOVATO, C.H. Odontologia hospitalar no Brasil. Uma visão geral. Rev Odontol UNESP. v.38, n.2, p.105-109, 2009.

GONÇALVES, C.L.; JUNIOR, M. F.S.; ANDRADE, L.S.; MICLOS, P.V. Odontologia hospitalar nos hospitais de grande porte da região metropolitana da Grande Vitória, Espírito Santo. Rev. Bras. Pesq. Saúde. V.16, n.1, p. 75-81, 2014.

LAGES, V. A.; NETO, J.M.M.; MELLO, P.M.V.C.; MENDES, R.F.; JÚNIOR, R.R.P. O efeito do tempo de internação hospitalar sobre a saúde bucal. Rev. Bras. Pesq. Saúde. V.16, n.2, p.3038, 2014.

LINO-JÚNIOR, H. L.; GABRIEL, M.; DARUGE - JÚNIOR, E.; SILVA, R. H. A. Ensino de Odontologia Legal no Brasil: um convite à reflexão. Rev ABENO. V.15, n.2, p.38-46, 2015.

LOBÃO, F.R.; DUARTE, M.V.; GUERREIRO, L.; PALAZZO, M.; ALMEIDA, P.; VARGAS, G.. O papel da Odontologia Intensiva. Academus Revista Científica da Saúde. V.1, n.3, 2016.

OGAWA, D.; HIGASI, M.S., CALDARELLI, P.G. Odontogeriatria nos Projetos Políticos Pedagógicos dos cursos de Odontologia do sul do Brasil. Rev ABENO. V.15, n.4, p.91-99, 
2015.

PINHEIRO, T.S.; ALMEIDA, T.F. A saúde bucal em pacientes de UTI. Rev Bahiana Odonto. v. 5, n.2, p.94-103, 2014.

ROCHA, A.L.; FERREIRA, E. Odontologia hospitalar: a atuação do cirurgião dentista em equipe multiprofissional na atenção terciária Hospital dentistry: the role of the dentist in multidisciplinary teams in tertiary care. Arq Odontol, v. 50, n. 4, p. 154-160, 2014.

SALDANHA, K.F.D.; COSTA, D.C.; PERES, P.I.; MOURA, M.O.; MASOCATTO, D.C.; JARDIM, E.C.G. A odontologia hospitalar: revisão. Arch Health Invest. V. 4, n.1, p. 58-68, 2015.

WAYAMA, M.T.; ARANEGA, A.M.; BASSI, A.P.F.; PONZONI, D.; GARCIA-JUNIOR, I.R.G. Grau de conhecimento dos cirurgiões-dentistas sobre Odontologia Hospitalar. Rev. Bras. Odontol. V.71, n.1, p. 48-52, 2014. 